\title{
WHISPERING GALLERY MODE LASERS IN ERBIUM/YTTERBIUM CODOPED PHOSPHATE GLASSES
}

\author{
Tran Thi Tam* \\ Faculty of Engineering Physics and Nano-Technology, \\ College of Technology, E3 144 Xuan Thuy Rd., Cau Giay, Hanoi, Vietnam \\ Dang Quoc Trung, Le Huu Minh, Tran Anh Vu, Do Ngoc Chung \\ Institute of Materials Science, 18 Hoang Quoc Viet Rd., Cau Giay, Hanoi, Vietnam

\section{Patrice Feron} \\ GIS "FOTON" Laboratoire d'Optronique (CNRS-UMR 6082) \\ ENSSAT 6 rue de Kerampont, 22300 Lannion, France
}

Received 27 November 2006

\begin{abstract}
The study of whispering gallery mode laser in $\mathrm{Er}^{3+} / \mathrm{Yb}^{3+}$ co-doped phosphate glass microsphere has been reported. The coupling was carried out by fiber half taper technique. The microsphere lasers have been studied under pumping at $980 \mathrm{~nm}$, to take full advantage of energy transfer effect from ion Ytterbium to ion Erbium. The observed lasing range extends from $1533 \mathrm{~nm}$ to $1612 \mathrm{~nm}$.
\end{abstract}

Keywords: Microsphere, whispering gallery modes, lasers, Erbium Ytterbium co-doped phosphate glass

\section{INTRODUCTION}

In the past decade, microcavities with its unique combination of strong temporal and spatial confinement of light have attracted interest for a large number of applications in either fundamental research from quantum electrodynamics (QED) to nonlinear optics, or in applied areas as photonics and optical communications [1,2]. Light in a dielectric microsphere can be guided to so called whispering gallery modes (WGMs). The mode volume in microsphere can be as low as a few hundred cubic wavelengths with quality factors $\boldsymbol{Q}$ above $10^{9}$ for fused silica. Rare earth-doped (Er or Nd) glass are the ideal subject for realizing these microspherical lasers. In a standard erbium-doped-fiber laser or amplifier, the length of the active fiber must be at least a few meters to obtain the enough absorption of the pump light. In the microsphere, the lasing effect has reached when the pump light excites microsphere fluorescent light along thin layer near equatorial to "whispering-gallery" waveguide modes that circulate by virtue of total internal reflection provided that the refraction index of the microsphere is higher than that of the surrounding. Inside the microsphere, the circulation of the "whispering-gallery" modes provides

* Corresponding author e-mail: ktlaser@ims.vast.ac.vn 
the necessary path length for absorption, thus making possible to reduce the laser threshold drastically. In this paper, we describe experimental results on microspheres laser oscillations of the $\mathrm{I}_{13 / 2} \rightarrow \mathrm{I}_{15 / 2}$ transition of $\mathrm{Er}^{3+}$ in phosphate glass obtained by using a tapered fiber for efficient coupling.

\section{EXPERIMENT}

\subsection{Fabrication of microspheres}

Microspheres have been produced from phosphate glass powders using a microwave plasma torch. The plasma is generated using a microwave supply with a nominal oscillator frequency of $2.4 \mathrm{GHz}$ and a maximum power of $2 \mathrm{~kW}$, the power and gas discharges can be adjusted to obtain optimal conditions to spheroidize fluoride or silicate glass. Argon is used as plasma gas and oxygen or nitrogen as sheath gas. Powders are axially injected and melt when passing through the plasma flame, superficial tension forces and then gave them their spherical form. Free spheres are collected a few ten centimeters lower, and then are glued at the tip of optical fibers of about $10 \mu \mathrm{m}$ to $30 \mu \mathrm{m}$ in diameter. The diameter of the spheres depends essentially on the powder size and varies from $10 \mu \mathrm{m}$ to $200 \mu \mathrm{m}$.

The used phosphate glass was an $\mathrm{Er}^{3+} / \mathrm{Yb}^{3+}$ co-doped phosphate glass (Schott IOG-2) doped with $2 \%$ weight of $\mathrm{Er}_{2} \mathrm{O}_{3}$ and co-doped with $3 \%$ weight $\mathrm{Yb}_{2} \mathrm{O}_{3}$. Photoluminescence spectroscopy, in the region of the $\mathrm{I}_{13 / 2} \rightarrow \mathrm{I}_{15 / 2}$ transition of $\mathrm{Er}^{3+}$ ion was performed using Cary 9000 spectrometer and the $980.8 \mathrm{~mm}$ line of a Ti:Sapphire laser as excitation source. The laser domain extended approximately from $1542 \mathrm{~nm}$ to $1605 \mathrm{~nm}$. The use of an $\mathrm{Er}^{3+} / \mathrm{Yb}^{3+}$ co-doped phosphate glass is associated to $975 \mathrm{~nm}$ pumping wavelength in order to populate the ${ }^{2} F_{5 / 2}$ metastable level of Ytterbium ions which transfer their energy to the neighboring Erbium ions by radiative and non-radiative ways [1]. To take full advantage of this excitation mechanism, we chose $975 \mathrm{~nm}$ among the different appropriate wavelengths for pumping Erbium/Ytterbium codoped glasses $(810,975$, and $1480 \mathrm{~nm})$. Also, the use of Ytterbium ions helps to avoid the unwanted side effects of a too high Erbium concentration (self pulsing etc.) used in experiment $\left(1.7 .10^{20}\right.$ ions $/ \mathrm{cm}^{3}$ for Erbium and $2.5 .10^{20}$ ions $/ \mathrm{cm}^{3}$ for Ytterbium).

\subsection{Excitation and receiving of WGMs}

Light may be coupled into and out of the microspheres by means of optical tunnel effect through evanescent field. For an efficient coupling of light into microspheres one must adjust the frequency of the excitation beam to a WGM resonance and align the excitation beam so that it also has an angular momentum matched with the angular momentum of that mode. The same one should be done to get WGMs signals out from microsphere. Many different techniques using high-index prisms, tapered fibers, angle polished fiber couplers or waveguides may be used for this purpose. Spheres must be set very close to the prism inside the evanescent field. In this experiment we use half-tapers for coupling light in and out for its relatively simple realization and mounting technique. The coupling can be achieved if we put the microspheres very close to the fiber half taper tip. The distance between microspheres and the fiber taper tip as well as an angle regarding microspheres's equator was controlled by micro positioning stages and/or with piezoelectric actuators.

\subsection{The experimental setup}

The experimental setup (see Fig. 1) was realized with standard fiber-optic components spliced or connected by APC connectors. Our experiments were performed with two direct fiber coupling schemes using half-tapered fibers: a) two separate half-tapers, one for coupling $980 \mathrm{~nm}$ pump in (Fig. 1a), the other for coupling signals out from the sphere, and b) using one single 120 
half-taper to couple both pump and laser fields in and out the micro spherical laser (Fig. 1b). The laser radiation coupled into the optical fiber $1.55 \mu \mathrm{m}$ is selected for output by a wavelength division demultiplexer (WDM). The pump source was fiber pigtailed SDL Laser Diode generating $976.1 \mathrm{~nm}$ radiation with the maximum $\mathrm{CW}$ power of $100 \mathrm{~mW}$. The maximum total power after WDM was $70 \mathrm{~mW}$. Although the optimum coupling for two wavelengths, $\lambda \sim 975$ $\mu \mathrm{m}$ for the pump and $\lambda \sim 1.55 \mu \mathrm{m}$ for the laser signal is not the same, we received good results even in b scheme (see Fig. 2). We produced the half tapers by chemical etching in HF or by heating and stretching a standard single mode at $1.55 \mu \mathrm{m}$ fiber until breaking it, using either $\mathrm{CO}_{2}$ laser or fusion optical splicing system. The fiber tip was tapered to $\sim 2 \mu \mathrm{m}$ in diameter. We fixed the co-doped $\mathrm{Er}^{3+} / \mathrm{Yb}^{3+}$ phosphate glass microspheres but mounted half tapers on $\mathrm{XYZ}$ Linear Micro translations with Rotation Stage. This setup allows to establish the equator region of the microspheres in the evanescent field surrounding the half taper and to adjust the acceptance angle (between the tip axis of the half taper and equatorial plane). The collected laser signal was analyzed with a $0.06 \mathrm{~nm}$ resolution Optical Spectrum Analyzer (OSA - Model: Agilent 86142B).

a)

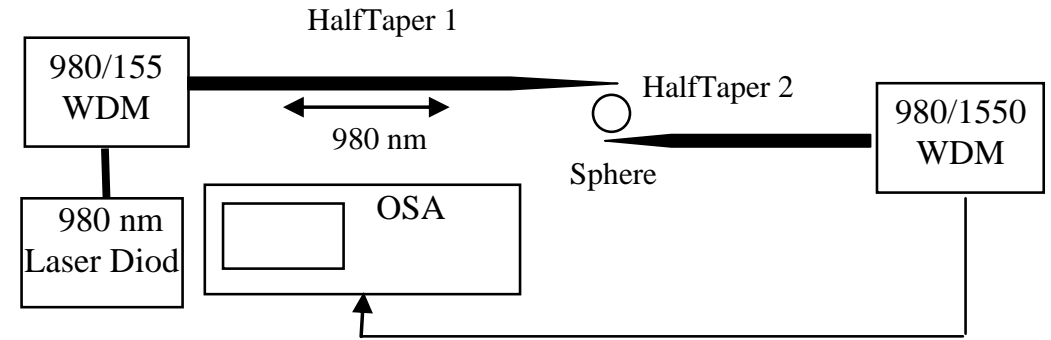

b)

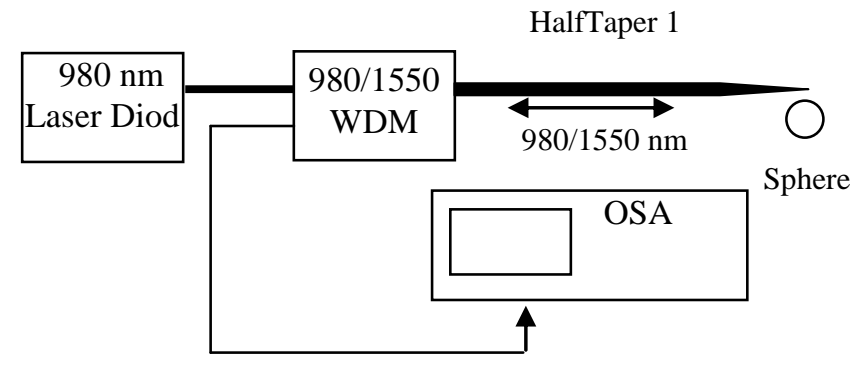

Fig. 1: The experimental setup: a) double half tapers; b) single half taper

\section{RESULTS}

The optical spectrum of the output signal from the sphere below the laser threshold shows the fluorescence intensity with a series of peaks. The estimation of the microsphere diameters through peaks distance in obtained fluorescence spectra gave results well matched with the ones received by optical method. When increasing the pump intensity we obtained a laser oscillation. Because of the difficulty in quantifying pump portion coupled in sphere, we controlled only the total output power of LD pump. The actual pump power at the tip was approximately $70 \%$ of that value. Figures 2 and 3 present several laser spectras from the microsphere of $140 \mu \mathrm{m}$ diameter under different total pump power levels.

In most cases a good coupling is obtained simultaneously for several lines with different wavelengths $\lambda$ so we have observed a multiline laser signal. The laser lines have been obtained in wide range from $1532.4 \mathrm{~nm}$ (Fig. 3a) to $1611.9 \mathrm{~nm}$ (Fig. 3b). This is a bit wider than the predicted ones (1542 $\mathrm{nm}$ to $1605 \mathrm{~nm}$ ) from spectroscopic data. The line width is limited by OSA resolution $(0.06 \mathrm{~nm})$. The peak power of the laser line is higher (the power reaches $3,5 \mu \mathrm{W}$ at 45 
$\mathrm{mW}$ of total pump power for the microsphere of diameter $90 \mu \mathrm{m}$ ) compared to the results when it had been pumped by $1480 \mathrm{~nm}$ [1]. The successful collecting of the laser emission from the microsphere depends on coupling half taper parameters. By adjusting the coupling parameters (the microsphere - half taper gap, the angle between the half taper and microsphere equator etc.) we can obtain laser in certain wavelength region between $1530 \mathrm{~nm}$ and $1620 \mathrm{~nm}$. However as a result of the mechanical imperfection of our mounting and translation system it presented the difficulty in getting a single laser mode and the definition of the lasing threshold. Figure 4 presents an example of selecting a single laser mode at $1534.4 \mathrm{~nm}$ from three lines by changing the acceptance angle. This has been achieved by choosing the coupling point in the half taper i.e. the distance from tip to acceptance point. Due to half taper non-constant diameter and consequently variable gap between the fiber and the microsphere, we can find the appropriate position to select one lasing mode. The electrostatic force which pulls in half tapers to microspheres also raised difficulties for getting the particular desired wavelength and made the collecting signal unstable. The laser emission can be extracted even when the half tapers are in contact with the sphere, though in this case we may get simultaneously a series of laser lines in a broader range (from $1557.8 \mathrm{~nm}$ up to $1611.9 \mathrm{~nm}$, see Fig. 2). In these cases we somehow managed to receive the single laser mode by varying the angle between half tapers axis and the equatorial plane of the sphere. The laser signal may be amplified for further application. When collecting the signal at "in contact" position, the small size of the half taper tip does not allow pumping with high power because it may burn the tip.

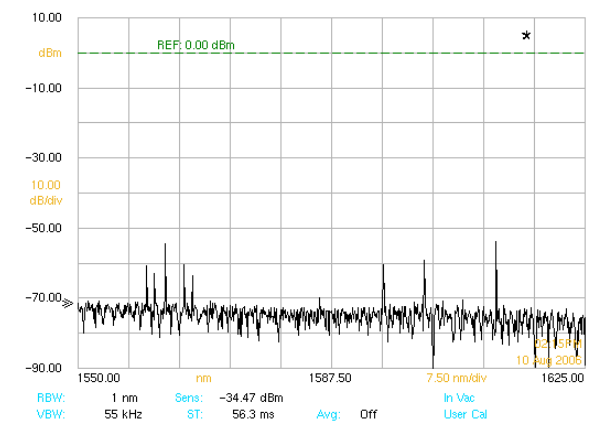

(a)

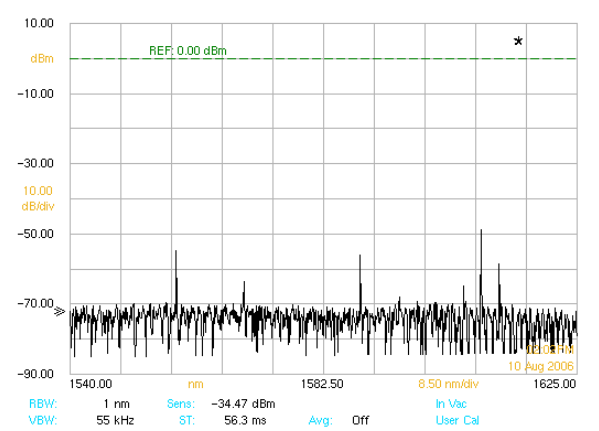

(b)

Fig. 2: The laser spectrum from microsphere of $140 \mu \mathrm{m}$ diameter, total pump: $45 \mathrm{~mW}$; (a) double half tapers; (b) single half taper

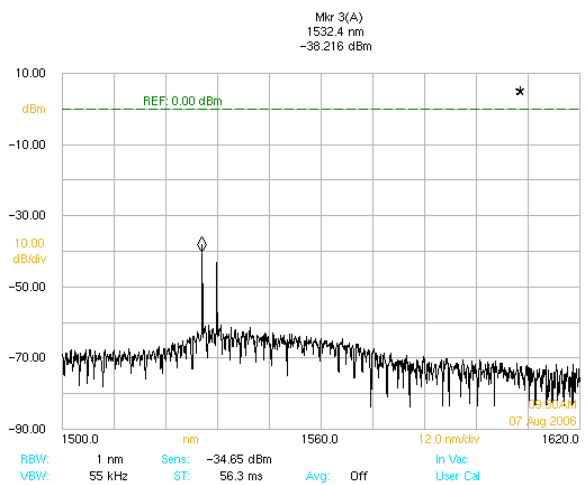

(a)

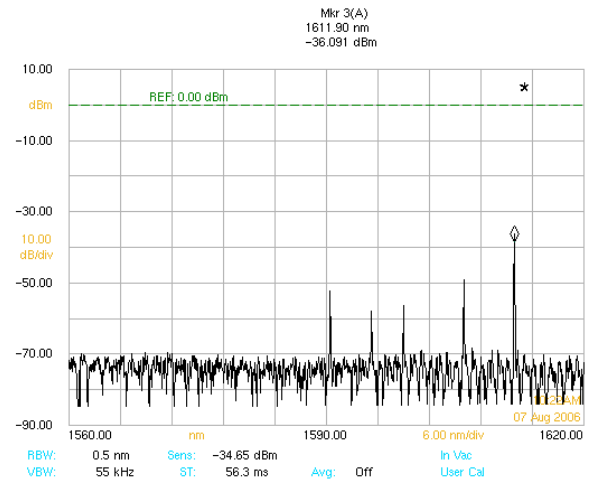

(b)

Fig. 3: The laser spectra from microsphere of $140 \mu \mathrm{m}$ diameter; (a) shortest observed wavelength $\lambda=1532.4 \mathrm{~nm}, p=150 \mathrm{nW}$; pump $25 \mathrm{~mW}$; (b) longest observed wavelength $\lambda=1611.90 \mathrm{~nm}, \mathrm{p}=246 \mathrm{nW}$; рump $65 \mathrm{~mW}$ 


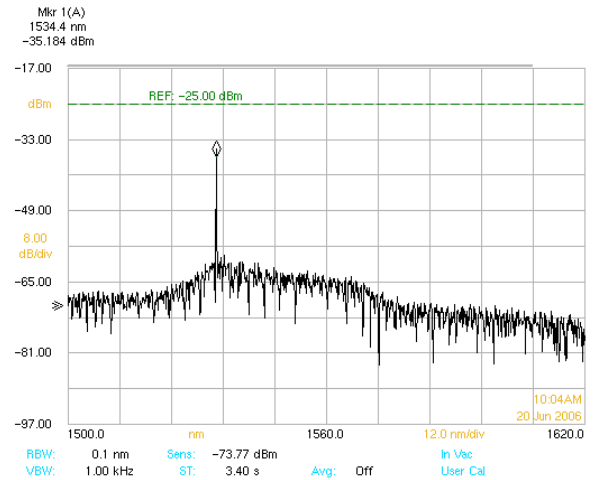

(a)

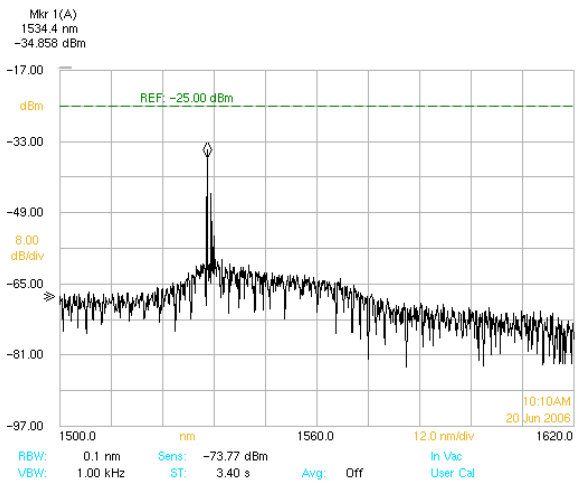

(b)

Fig. 4: The laser spectrum from microsphere of 140 um diameter, pump $40 \mathrm{~mW}$; (a) three lines; (b) single line, power $300 \mathrm{nW}$

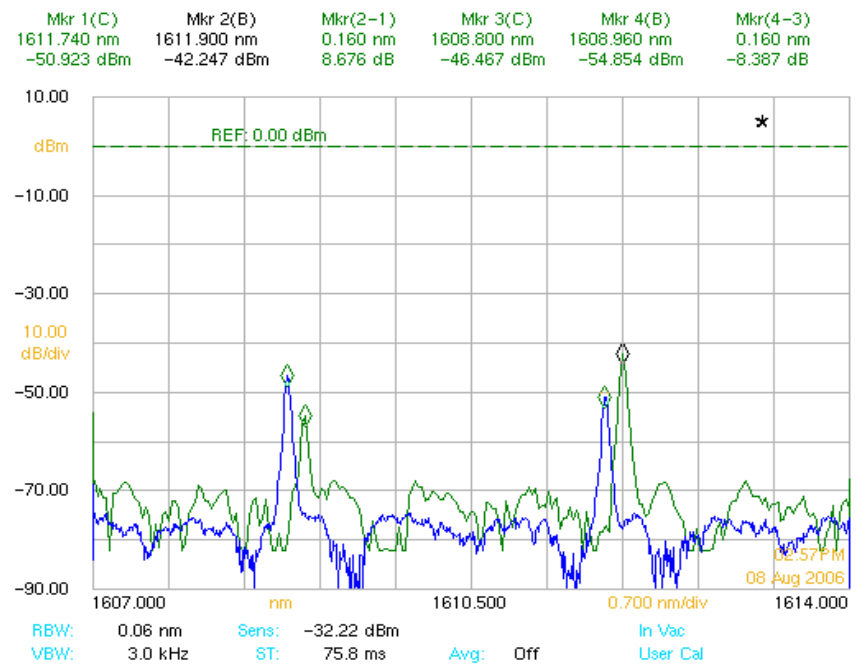

Fig. 5: The wavelength shift in laser spectrum, microsphere of $140 \mu \mathrm{m}$ diameter, pump: $25 \mathrm{~mW}$ and $60 \mathrm{~mW}$

When increasing the pump power beside newly emerged laser lines we also observed a small red shift in the wavelength of WGMs. This red-shift effect on the wavelength of laser spectra when the pump power is increased was previously experimentally observed in Er/Yb phosphate microchip laser [1] and Er:ZBLALiP microspherical laser [3] and explained by a simple model based on thermal effects. The typical result of the laser spectra analyzed by an Optical Spectrum Analyzer with a resolution of $0.060 \mathrm{~nm}$ is illustrated in Fig. 5. The two wavelengths at 1608.80 $\mathrm{nm}$ and $1611.74 \mathrm{~nm}$ when the pump power intensity is $25 \mathrm{~mW}$ shift further to $1608.96 \mathrm{~nm}$ and $1611.90 \mathrm{~nm}$, respectively, when the total pump power was increased to $60 \mathrm{~mW}$. Similar redshift behaviours have also been observed for spheres of other size. Except several new lines emerged under strong pump, all existing WGMs shifted by $0.16 \mathrm{~nm}$ towards longer wavelength under the total power domain increasing from 25 to 60. This red shift phenomenon is explained as thermal effect [5]. The phonons inside the active microspherical laser cavity associate with the non radiative decay between the manifolds of Erbium ions, and between the intra-Stark levels of the laser manifolds, thus create the thermal deposition and heat the microsphere. An increase of cavity temperature $\Delta T$ results in both an expansion $\Delta d$ of the microsphere cavity 
length and a change of index of refraction $\Delta N$. Both changes then affect the lasing condition and the wavelength shift $\Delta \lambda$ of every WGM as the cavity temperature rises by $\Delta T$ can be written as [5] $\Delta \lambda=\lambda \cdot(1 / N \cdot \partial N / \partial T+1 / d \cdot \partial d / \partial T) \cdot \Delta T$

Here $N$ and $d$ are constant values which are referenced to the room temperature, or more precisely, to the temperature corresponding to the threshold for oscillation. $\partial N / \partial T$ and $\partial d / \partial T$ are change ratio with respect to temperature for the index of refraction and thermal expansion of the glass.

\section{CONCLUSION}

The Microsphere Whispering Gallery Mode lasers have been observed in $\mathrm{Er}^{3+} / \mathrm{Yb}^{3+}$ co-doped phosphate glass under $976 \mathrm{~nm}$ pumping, to take the full advantage of the energy transfer effect from ion Ytterbium to ion Erbium. The coupling was carried out by fiber half taper technique in two schemes, both gave good results. The single laser line power may reach $150 \mathrm{nW}$ with only $25 \mathrm{~mW}$ total pump power, and laser range extends from $1532.4 \mathrm{~nm}$ to $1611.9 \mathrm{~nm}$. The laser spectra analyzed by OSA showed the red shift effect which associate with the thermal effect occurred inside the sphere under strong pump.

\section{ACKNOWLEDGEMENT}

These works are supported by National Basic Research Program KT-04 and are the collaboration between IMS, College of Technology, Vietnam and CNRS, France. The authors thank CNR-IFAC, "Nello Carrara" Institute of Applied Physics, 50127 Firenze, Italy for supplying the $\mathrm{Er}^{3+} / \mathrm{Yb}^{3+}$ co-doped phosphate glass. The authors would like to express the support of subject of 410606 number at KT04 basic science program.

\section{REFERENCES}

1. Lefevre-Seguin, V. and Haroche, S. (1997), Mat. Sci. and Engin., vol. B 48, p. 53.

2. Little, B.E., Lame, J.P., Lim, D.R., Haus, H.A., Kimerling, L.C., and Chu, S.T. (2000), Opt. Lett., vol. 25, p. 73.

3. Riseberg, A. and Moos, H.W. (1968), Phys.Rev., vol. 174, p. 429.

4. Patrice Feron (2004), Annales de la Fondation Louis de Broglie, vol. 29(1-2), p. 317.

5. Cai, Z.P., Chardon, A., Xu, H.Y., Feron, P., and Stephan, G.M. (2002), Opt. Comm., vol. 203, p. 301. 